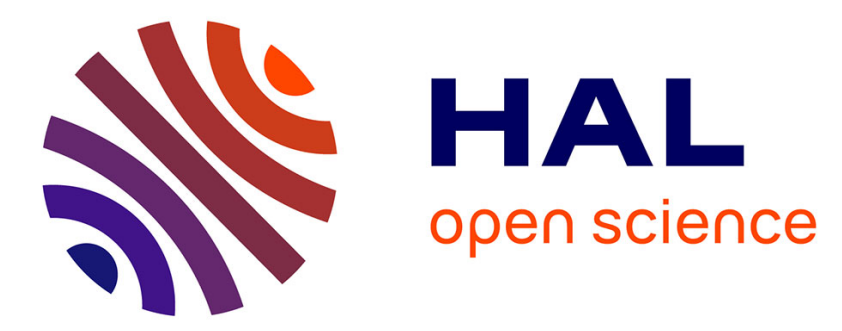

\title{
Les infections intra-mammaires chez la vache laitière dans l'enquête écopathologique Bretagne
}

B. Faye, Nelly Dorr, Françoise Lescourret, J. Barnouin, Michelle Chassagne

\section{To cite this version:}

B. Faye, Nelly Dorr, Françoise Lescourret, J. Barnouin, Michelle Chassagne. Les infections intramammaires chez la vache laitière dans l'enquête écopathologique Bretagne. Productions Animales, 1994, 7 (1), pp.55-65. hal-00896076

\section{HAL Id: hal-00896076 https://hal.science/hal-00896076}

Submitted on 1 Jan 1994

HAL is a multi-disciplinary open access archive for the deposit and dissemination of scientific research documents, whether they are published or not. The documents may come from teaching and research institutions in France or abroad, or from public or private research centers.
L'archive ouverte pluridisciplinaire HAL, est destinée au dépôt et à la diffusion de documents scientifiques de niveau recherche, publiés ou non, émanant des établissements d'enseignement et de recherche français ou étrangers, des laboratoires publics ou privés. 
INRA Prod. Anim., $1994,7(1), 55-65$
B. FAYE, Nelly DORR,

Françoise LESCOURRET,

J. BARNOUIN, Michelle CHASSAGNE

INRA Laboratoire d'Ecopathologie

Theix 63122 St Genès-Champanelle
Les infections intra-mammaires chez la vache laitière dans l'enquête écopathologique Bretagne

taines bactéries peuvent être pathogènes pour l'homme lors de consommation de lait cru ou de produits laitiers à base de lait cru), mais aussi des problèmes technologiques : modification des taux, difficultés à la transformation du lait. Enfin, la mise en place du paiement multicritères du lait incite les producteurs à privilégier l'option qualité, les laits les plus mal classés accusant une pénalisation parfois considérable. La notion de qualité hygiénique peut s'aborder de diverses manières, mais, en tout état de cause, la mamelle reste l'une des principales sources de contamination. La connaissance détaillée de la fréquence actuelle des différents germes responsables d'infections mammaires et intramammaires présente un grand intérêt pour la définition de stratégies de lutte appropriées, l'expérience montrant que les facteurs de risque ne sont pas les mêmes selon la nature des germes pathogènes en cause (Schukken 1990, Faye et al 1993). Les programmes de contrôle appliqués actuellement sont d'ailleurs définis en fonction de l'épidémiologie des espèces bactériennes responsables (Poutrel 1985).

Cet article expose les résultats descriptifs concernant les infections intra mammaires dans le cadre de l'enquête écopathologique Bretagne (Faye et Barnouin 1987).

Quelques facteurs de variation et les relations avec d'autres critères qualifiant l'atteinte de la mamelle (mammites cliniques, concentration cellulaire dans le lait) sont également abordés.

\section{1 / Conduite de l'étude}

Les données proviennent des informations recueillies pendant 4 années auprès de 47 exploitations de la région Bretagne. La base de données constituée à partir de ces informations (Lescourret et al 1993) concerne 4129 vaches représentant 8945 lactations. Outre les analyses microbiologiques du lait individuel des vaches, nous disposons de toutes les informations relatives aux condi- 
tions d'environnement et aux pratiques d'élevage. Le but de l'étude est de mettre en évidence les facteurs de risque collectifs (pratiques d'élevage, conditions d'environnement) et individuels (race, rang de lactation, niveau de production...) qui influent sur les mammites cliniques, subcliniques et les infections intramammaires. Nous nous limitons dans le cadre de cet article à l'étude de quelques facteurs de variation liés au temps (saison, rang de lactation, stade physiologique, année) et à l'espace (département).

\section{Les prélèvements de lait}

Des prélèvements de lait ont été effectués sur toutes les vaches suivies dans le cadre de cette étude en début de lactation (au cours des 7 premières semaines) par des techniciens des services vétérinaires du Finistère, du Morbihan et d'Ille et Vilaine) ; soit au total 7852 prélèvements répartis de la manière suivante : 1022 (semaine 1), 1288 (semaine 2), 1255 (semaine 3), 1223 (semaine 4), 1251 (semaine 5), 1170 (semaine 6) et 643 (semaine 7). Les prélèvements ont été réalisés avant la traite du matin, après nettoyage de l'extrémité des trayons, extraction des premiers jets et désinfection à l'alcool à $70^{\circ}$ durant 30 secondes. Le lait a été prélevé à chaque trayon pour limiter les risques de contamination. Les analyses bactériologiques ont été réalisées dans les laboratoires départementaux sur le lait de mélange des 4 trayons, selon les méthodes classiques. L'analyse sur les laits de mélange conduit à une perte d'information, mais ce choix a été guidé essentiellement pour des raisons de coût. Par ailleurs, notre objectif étant d'avoir des indicateurs épidémiologiques et non un diagnostic microbiologique des mammites, nous avons opté pour une simplification du protocole d'analyse.

\section{Informations sanitaires}

Les infections mammaires cliniques ont été répertoriées par l'éleveur et/ou son vétérinaire sur un agenda transmis aux techniciens des services vétérinaires lors de leur passage mensuel dans les exploitations. Selon la nature des symptômes relevés, 6 types de mammites ont été identifiés :

- mammite caractérisée par une modification apparente du lait (grumeaux, couleur ...). Ce symptôme est codé MELA dans la base de données ;

- mammite caractérisée par une inflammation d'au moins un quartier (rougeur + chaleur + douleur). Ce symptôme est codé MEIN ;

- mammite présentant simultanément les caractères précédents (lait modifié + inflammation de la mamelle). Ce symptôme est codé MELM ;

- mammite dont les caractéristiques cliniques n'ont pas été spécifiées (code MEXX) ;

- mammite s'accompagnant de signes généraux (hyperthermie, abattement) nécessitant un traitement par voie générale (code MEFI) ;
- enfin, mammite grangréneuse, codée MEGA, d'incidence très rare.

Par ailleurs, la localisation des infections mammaires a également été relevée : quartier avant gauche (1), avant droit (2), arrière gauche (3) et arrière droit (4). Les récidives sur le même quartier, avec le même code, n'ont pas été prises en compte dès lors qu'elles survenaient moins de 3 mois après l'atteinte précédente. Disposant enfin des données du contrôle laitier, les relevés mensuels des concentrations cellulaires individuelles ont été enregistrés dans la base de données.

\section{Traitement statistique}

L'étude présentée ici est descriptive. Seuls les tests d'indépendance du Chi2 sur des tableaux de contingence ont été utilisés. Après extraction des données par le langage SQL, la gestion des tableaux de données a été opérée avec l'aide du logiciel SAS.

\section{2 / Résultats}

\section{1 / Population bactérienne dans les infections intramammaires}

Au total, 18 espèces de bactéries et levures ont été isolées dans les 4164 prélèvements qui se sont avérés non stériles, soit dans $53 \%$ des cas. Plusieurs germes pouvant être isolés sur un même échantillon de lait, on relève au total 4327 germes isolés (tableau 1). En début d'étude et dans un département, un nombre non négligeable de prélèvements $(7,9 \%)$ contenait des germes qualifiés de contamination par le laboratoire. Ces prélèvements ont été écartés des analyses ultérieures.

Pour certaines analyses, les germes ont été regroupés en trois catégories:

- germes pathogènes mineurs (GMIN) caractérisés par un faible pouvoir pathogène: $A B$, $\mathrm{AS}$ et $\mathrm{BO}$;

- germes pathogènes majeurs (GMAJ) qui ont un pouvoir pathogène important et provoquent des mammites cliniques et une montée des concentrations cellulaires : DI, EC, ST, UB et $\mathrm{AC}$;

- germes pathogènes rares (GRAR) qui peuvent être majeurs (AE, AG, CA, KP, LM, ND, $\mathrm{PY}, \mathrm{PS}$ ) ou non (CE).

\section{2 / Présence simultanée de plusieurs germes dans le lait de mélange}

Sur les 4164 prélèvements non-stériles, 660 contiennent deux germes associés (soit 15,8\% des prélèvements) et 61 contiennent trois germes associés $(1,6 \%$ des prélèvements nonstériles). Bien que l'indépendance des quartiers de la mamelle de la vache ne permette pas de se prononcer sur la réalité des associations de germes à l'intérieur d'un même quartier, l'analyse étant faite sur le lait de mélange, 
Tableau 1. Répartition des différents germes isolés dans 7852 prélèvements de lait de traite chez la vache laitière en début de lactation.

\begin{tabular}{|l|l|c|c|}
\hline Code & Germe isolé & $\begin{array}{c}\text { Nombre } \\
\text { d'échantillons }\end{array}$ & $\begin{array}{c}\text { \% du nombre total } \\
\text { de prélèvements }\end{array}$ \\
\hline AS & Autres staphylocoques & 2188 & 27,87 \\
ST & Staphylococcus aureus & 553 & 7,03 \\
AC & Autres streptocoques & 513 & 6,53 \\
UB & Streptococcus uberis & 420 & 5,35 \\
AB & Autres bacillus & 276 & 3,52 \\
BO & Corynebacterium bovis & 147 & 1,87 \\
EC & Escherichia coli & 106 & 1,35 \\
DI & Streptococcus dysgalactiae & 73 & 0,92 \\
CA & Candida albicans & 17 & 0,22 \\
PY & Corynebacterium pyogenes & 12 & 0,15 \\
AG & Streptococcus agalactiae & 10 & 0,13 \\
PS & Pseudomonas sp & 6 & 0,08 \\
NO & Nocardia sp & 2 & 0,02 \\
AE & Autres entérobactéries & 1 & 0,01 \\
CE & Bacillus cereus & 1 & 0,01 \\
KP & Klebsiella pneumoniae & 1 & 0,01 \\
LM & Listeria monocytogenes & 1 & 0,01 \\
CO & Germes de contamination & 619 & 7,87 \\
\hline
\end{tabular}

Tableau 2. Fréquence d'association (en \%) des germes pathogènes avec un ou deux autres germes pathogènes dans le même prélèvement de lait.

\begin{tabular}{|l|c|c|}
\hline Germe pathogène & \multicolumn{2}{|c|}{$\begin{array}{c}\text { Fréquence d'association avec } \\
\text { 1 germe }\end{array}$} \\
2 germes \\
\hline Autres bacillus & 46,0 & 11,5 \\
Autres streptocoques & 44,0 & 7,0 \\
Escherichia coli & 42,8 & 8,4 \\
Streptococcus uberis & 31,9 & 4,2 \\
Staphylococcus aureus & 27,0 & 3,4 \\
Corynebacterium bovis & 23,8 & 0,6 \\
Autres staphylocoques & 21,3 & 2,4 \\
\hline
\end{tabular}

Tableau 3. Tableau d'occurence des associations de 3 germes différents dans les infections intramammaires.

\begin{tabular}{|c|c|c|c|c|c|c|}
\hline AB-AC-ST & 1 & & & & & \\
\hline AB-UB-ST & 1 & & & & & \\
\hline AC-AS-CA & 1 & & & & & \\
\hline AG-AS-EC & 1 & & & & & \\
\hline AS-AC-BO & 1 & & & & & \\
\hline AS-ST-EC & 1 & & & & & \\
\hline AS-CA-UB & 1 & & & & & \\
\hline AB-AS-PY & & 2 & & & & \\
\hline AB-ST-DI & & 2 & & & & \\
\hline DI-UB-ST & & 2 & & & & \\
\hline AS-UB-AC & & 2 & & & & \\
\hline AS-ST-UB & & 2 & & & & \\
\hline AC-ST-UB & & 2 & & & & \\
\hline EC-AS-AC & & 2 & & & & \\
\hline AB-AS-S'T & & & 3 & & & \\
\hline AS-UB-EC & & & 3 & & & \\
\hline AS-AB-UB & & & & 4 & & \\
\hline AC-AS-ST & & & & & 5 & \\
\hline AB-AC-AS & & & & & & 19 \\
\hline
\end{tabular}

la présence simultanée de plusieurs germes dans la mamelle peut constituer néanmoins un indicateur de la diversité du microbisme à tropisme mammaire dans un élevage donné à un moment donné. Les associations les plus fréquentes concernent les germes pathogènes mineurs entre eux. Quatre bactéries (au demeurant très rarement présentes) sont toujours identifiées sans association avec d'autres germes : $\mathrm{AE}$ (autres enterobactéries), $\mathrm{CE}$ (Bacillus Cereus), KP (Klebsiella pneumoniae) LM (Listeria monocytogenes). Les fréquences d'association avec 1 ou 2 autres germes sont répertoriées dans le tableau 2. Parmi les germes rares, PY (Corynebacterium pyogemes) et AG (Streptococcus agalactiae) sont les plus fréquemment associés (respectivement dans 75 et $70 \%$ des prélèvements les concernant). Lorsqu'un prélèvement contient 3 germes, la combinaison la plus fréquente est $\mathrm{AB}, \mathrm{AC}, \mathrm{AS}$ (tableau 3). Parmi les germes pathogènes majeurs, c'est Escherichia coli qui est le plus souvent associé à d'autres germes, plus généralement d'ailleurs avec des germes mineurs (AS) ou avec des streptocoques (AC).

\section{3 / Quantité de germes}

La quantité des germes isolés dans chaque prélèvement a été prise en compte en attribuant une classe de quantité reflétant le nombre de colonies isolées sur les cultures : 1 (nombre de colonies faible), 2 (moyennement abondant), 3 (très abondant). Quelques prélèvements étaient non renseignés. Bien que la concentration bactérienne dans le lait varie beaucoup au cours du déroulement de l'infection mammaire, l'abondance des colonies peut représenter une indication intéressante au niveau épidémiologique. Les classes de quantité 1 sont globalement les plus représentées : $54 \%$ des prélèvements contenant un germe pathogène mineur, $42 \%$ un germe pathogène 
Figure 1. Répartition des fréquences des différents germes par classe de quantité (1, 2 ou 3).

Fréquence (\%)

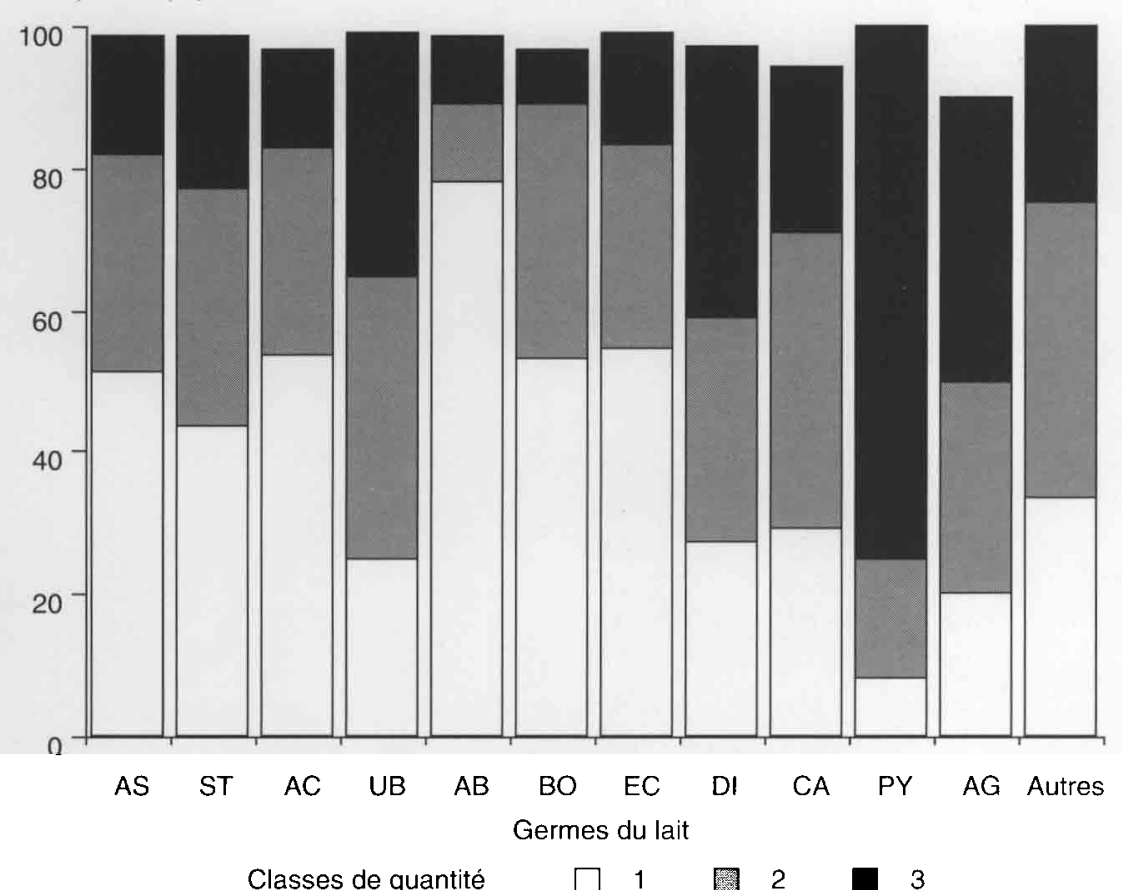

majeur et $23 \%$ un germe rare. Par type de germes, les bactéries les moins abondantes dans un prélèvement donné sont les bacillus (78\% des prélèvements contenant un bacillus sont de quantité 1). Les classes de quantité 2 se rencontrent surtout avec Candida albicans (41\% des prélèvements) et Streptococcus uberis ( $40 \%$ des prélèvements) ainsi que les germes rares entrant dans la catégorie "autres" (figure 1). Enfin, les colonies les plus abondantes (classe de quantité 3 ) se rencontrent plutôt chez les germes pathogènes majeurs (38\% des prélèvements contenant un Streptococcus dysgalactiae) ou rares (40\% pour Streptococcus agalactiae, $75 \%$ pour Corynebacterium pyogenes).

\section{4 / Persistance des infections intramammaires}

La quasi-totalité des vaches dans notre échantillon a subi un traitement anti-infectieux au tarissement. Pourtant, dans un certain nombre de cas, des réoccurences d'infections intramammaires sont observées.
Tableau 4. Persistance des infections intramammaires (en \%) à la lactation $n+1$ selon les germes isolés à la lactation n pour les vaches prélevées au cours de 2 lactations successives.

\begin{tabular}{|l|c|c|c|c|}
\hline & \multicolumn{4}{|c|}{ Lactation n+1 } \\
Lactation n & \multicolumn{1}{|c|}{ GMIN } & \multicolumn{1}{c|}{ GMAJ } & \multicolumn{1}{c|}{ GRAR } & ABSG \\
\hline GMIN & 38,2 & 20,5 & 1,4 & 39,9 \\
GMAJ & 31,8 & 29,5 & 1,3 & 37,4 \\
GRAR & 28,5 & 57,1 & 0,0 & 14,4 \\
ABSG & 24,6 & 14,0 & 0,6 & 60,8 \\
\hline
\end{tabular}

GMIN : germes mineurs, GMAJ : germes majeurs,

GRAR : germes rares, ABSG : absence de germes

Parmi les 1025 vaches qui ont subi deux prélèvements de lait au cours de 2 lactations successives, $38,2 \%$ de celles qui ont présenté un germe pathogène mineur à la lactation $n$ ont récidivé à la lactation $\mathrm{n}+1$. Ce taux est de $29,5 \%$ pour les germes majeurs, $0 \%$ pour les germes rares et $60,8 \%$ pour les vaches sans germes (tableau 4). Les infections dues aux streptocoques $(\mathrm{AC})$ lors de la lactation $\mathrm{n}$ prédisposent à des récidives avec des staphylocoques (AS) dans $43,8 \%$ des cas lors de la lactation $n+1$. Ce taux n'est que de $38,0 \%$ dans le sens $A B(n) \rightarrow A S(n+1)$ et $29,3 \%$ dans le sens $\mathrm{AS}(n) \rightarrow \mathrm{AS}(n+1)$. Globalement, les germes mineurs prédisposent peu à une réinfection par des germes majeurs à la lactation suivante $(20,5 \%)$. Seules les infections dues aux bacillus sont suivies par une sensible prédominance des infections à $S$. aureus à la lactation suivante $(16,6 \%)$. A l'inverse, les infections intramammaires dues à des germes majeurs sont plus fréquemment suivies d'infections dues à des germes mineurs à la lactation suivante (dans $31,8 \%$ des cas), la prédisposition la plus importante étant due à $S$. uberis suivi dans $28,0 \%$ des cas par un isolement de AS au cours de la lactation suivante. En revanche, $E$. coli est rarement associé à des germes mineurs au cours de la lactation suivante (moins de $10 \%$ ). Parmi les germes majeurs, la répétabilité la plus importante est observée avec $S$. aureus $(16,2 \%)$. Les germes rares prédisposent plutôt à des infections par des germes majeurs $(57,1 \%)$ mais les effectifs concernés sont très faibles. 648 vaches ont subi 3 prélèvements au cours de 3 lactations successives. Globalement, on observe les mêmes tendances (tableau 5 ). La persistance des infections intramammaires dues à des germes mineurs reste élevée (supérieure à $40 \%$ ) particulièrement pour les staphylocoques (AS), contrairement aux germes

Tableau 5. Persistance des infections intramammaires (en \%) aux lactations $n+1$ et $n+2$ selon les germes isolés à la lactation n pour les vaches prélevées au cours de 3 lactations successives.

\begin{tabular}{|l|c|c|c|c|c|c|c|c|}
\hline & \multicolumn{4}{|c|}{ Lactation n+1 } & \multicolumn{4}{c|}{ Lactation n+2 } \\
Lactation n & GMIN & \multicolumn{1}{|c|}{ GMAJ } & \multicolumn{1}{c|}{ GRAR } & ABSG & GMIN & GMAJ & GRAR & ABSG \\
\hline GMIN & 45,6 & 20,5 & 0 & 33,9 & 41,5 & 20,5 & 0 & 38,0 \\
GMAJ & 36,8 & 27,4 & 0 & 35,8 & 41,2 & 23,9 & 1,2 & 33,7 \\
GRAR & 25,0 & 75,0 & 0 & - & 75,0 & 12,5 & 0 & 12,5 \\
ABSG & 31,0 & 12,7 & 0 & 56,3 & 28,1 & 16,0 & 0,7 & 55,2 \\
\hline
\end{tabular}


majeurs (répétabilité de l'ordre de $20 \%$ ). Enfin, 485 vaches ont subi 4 prélèvements de lait. On observe des valeurs similaires aux précédentes, avec une tendance générale à la baisse des taux de répétabilité au cours des lactations successives : $49,6-35,3-36,7 \%$ pour les germes mineurs, $31,6-24,7-26,7 \%$ pour les germes majeurs.

\section{5 / Facteurs de variation des infections intramammaires}

\section{a / la semaine de lactation}

Les prélèvements ont été effectués en début de lactation. Cependant, du fait du passage mensuel des techniciens, il subsiste un écart date de vêlage - date de prélèvement qui varie d'un animal à l'autre (de 1 à 7 semaines). Cet écart pouvant introduire un éventuel biais, il importe de l'estimer. En fait, il n'y a pas d'effet significatif de la semaine de prélèvement, ni sur les germes mineurs, ni sur les germes majeurs (figure 2), bien que Staphylococcus aureus ait tendance à être plus fréquent avec l'augmentation de l'écart vêlage - prélèvement, à l'inverse de Streptococcus uberis qui tend à diminuer. Cependant, on observe une augmentation faible, mais significative des fréquences de Corynebacterium bovis en fonction du stade de lactation $(\mathrm{P}<0,05)$, celles-ci passant de 0,8 à $3,7 \%$ des prélèvements. Globalement, nous observons une tendance à l'augmentation de la fréquence des infections intramammaires au cours des sept premières semaines de lactation (tableau 6).

\section{$b$ / le rang de lactation}

Afin de disposer d'effectifs suffisants, les rangs de lactation 8 et supérieurs ont été regroupés. L'évolution des fréquences d'infections intramammaires par les germes mineurs n'indique pas d'effet signigicatif du rang de lactation. Les primipares sont plus affectées $(>35 \%)$ que les animaux de rang supérieur (figure 3). La fréquence des infections intramammaires due à des germes majeurs augmente avec le rang de lactation $(\mathrm{P}<0,001)$. Le taux observé chez les femelles de rang 8 et plus $(35,5 \%)$ est plus du double de celui des primipares $(17,6 \%)$. Cette évolution dans le temps est notable pour tous les types de germes majeurs, mais plus particulièrement pour $S$. uberis. Les Streptocoques et $E$. coli

Tableau 6. Fréquence des prélèvements stériles en fonction du stade de lactation.

\begin{tabular}{|c|c|c|}
\hline $\begin{array}{l}\text { Semaine } \\
\text { de lactation }\end{array}$ & $\mathbf{n}$ & Stériles $(\%)$ \\
\hline 1 & 1024 & 51,3 \\
2 & 1288 & 48,7 \\
3 & 1255 & 45,8 \\
4 & 1223 & 45,8 \\
5 & 1251 & 45,5 \\
6 & 1170 & 44,9 \\
7 & 643 & 45,7 \\
\hline
\end{tabular}

Figure 2. Evolution de la fréquence des infections intramammaires en fonction du stade physiologique, selon les types de germes.

Fréquence hebdomadaire

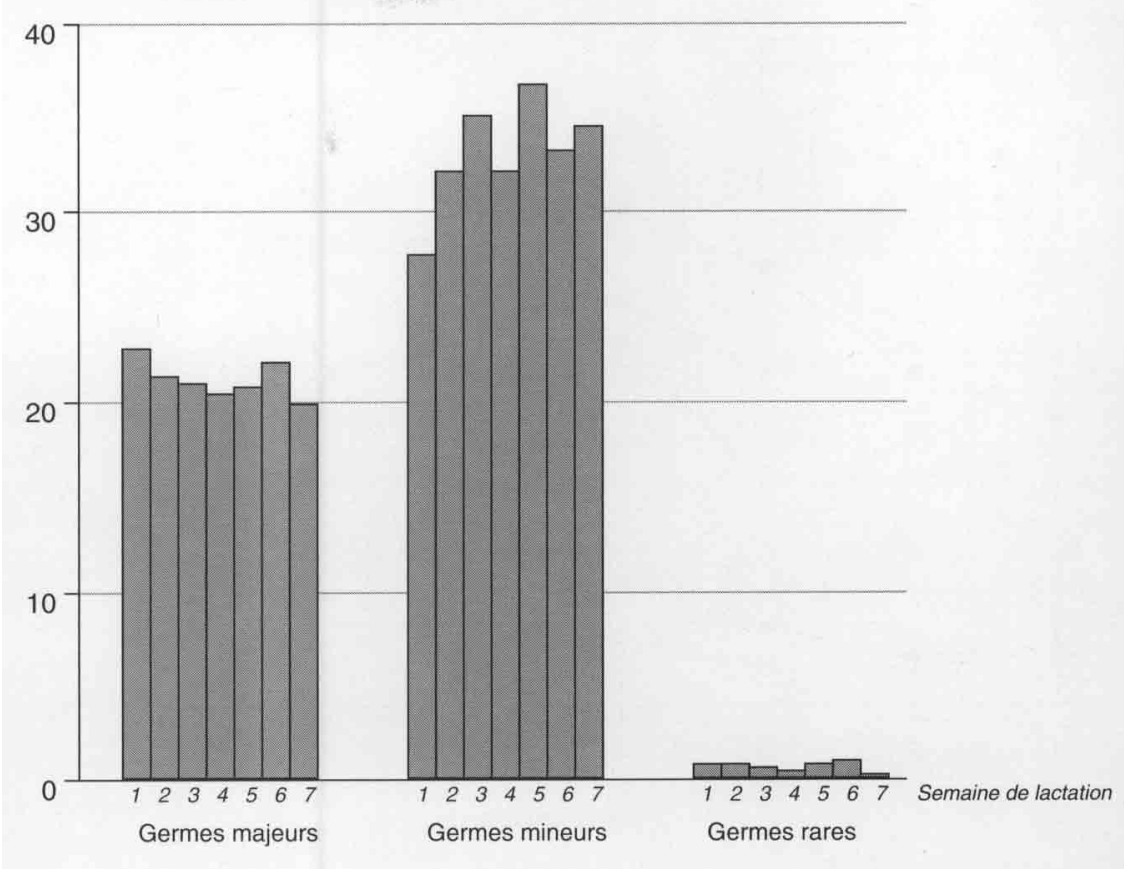

Figure 3. Evolution des fréquences d'infections intramammaires selon le rang de lactation.

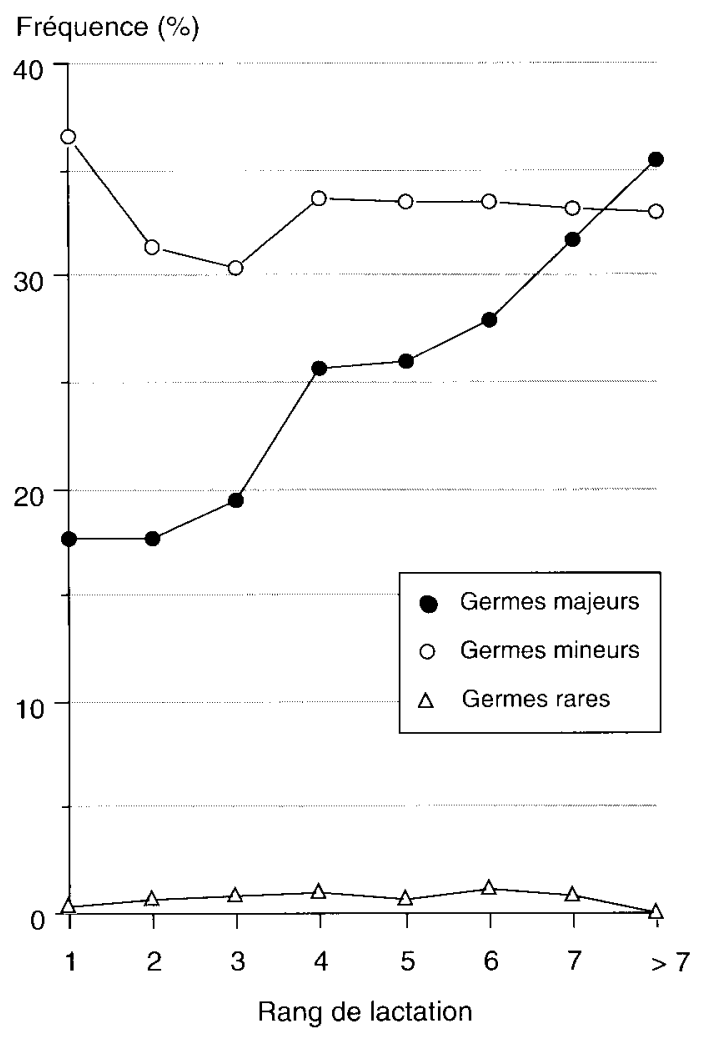

sont plus fréquemment isolés chez les vaches de rang de lactation élevé (figure 4). L'évolution des taux d'infections intramammaires dues à des germes rares n'est pas significative, on observe néanmoins une tendance à l'augmentation des taux jusqu'à la $6^{\text {eme }}$ lactation. 
Figure 4. Evolution des fréquences d'infections intramammaires selon le rang de lactation pour les germes pathogènes majeurs.

Fréquence (\%)

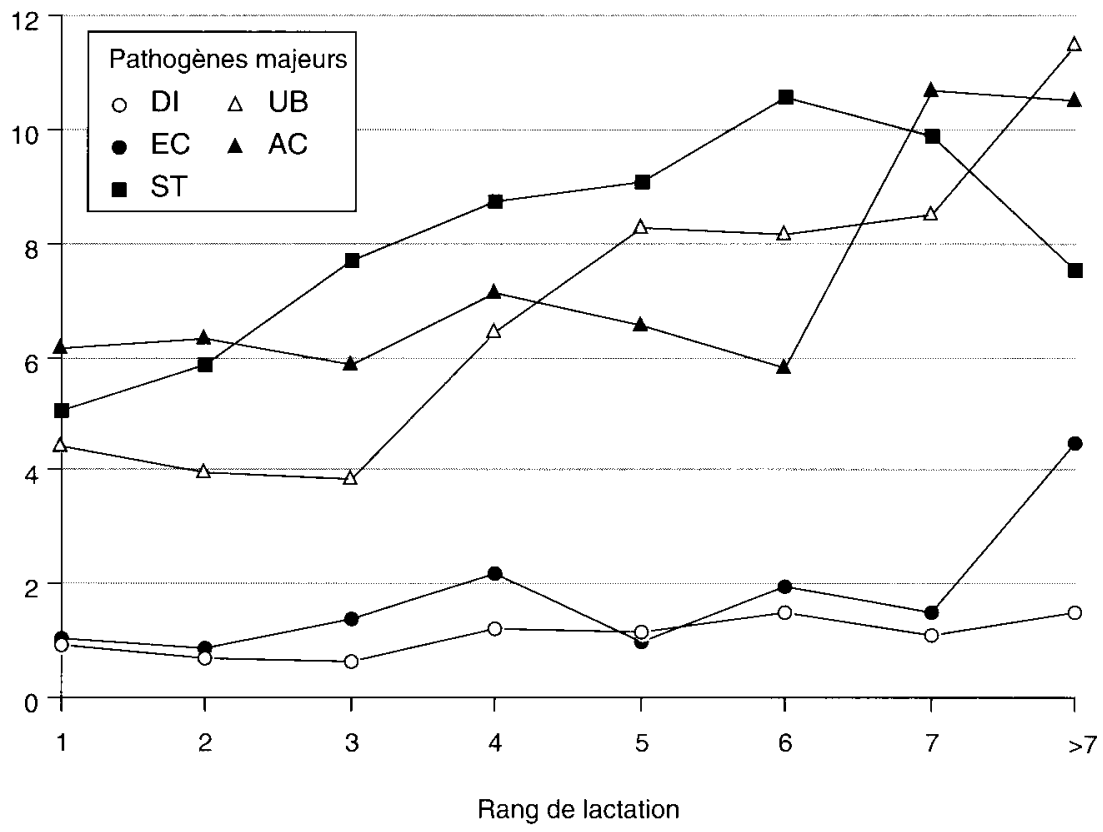

\section{c / le mois de prélèvement (effet saisonnier)}

Les observations à l'échelle de la semaine permettent de considérer que l'effet saisonnier éventuel est indépendant d'un effet "stade de lactation" à l'exception notable de $C$. bovis. D'un point de vue général, on observe des variations mensuelles signifivatives $(\mathrm{P}<0,001)$ indiquant un effet saisonnier important quel que soit ce type de germes. Les infections intramammaires dues aux germes mineurs passent par un maximum en octobre-novembre et un minimum en juillet-août. Cette période de faible incidence est décalée en septembreoctobre pour les germes majeurs. Si la période de stabulation hivernale est globalement défavorable, l'incidence des infections à des germes majeurs reste élevée jusqu'en juin. Ce pic du mois de juin est d'ailleurs observable pour la plupart des germes pathogènes (ST, UB, EC). La chute des incidences à l'automne est particulièrement accentuée pour $S$. uberis (figure 5). Les germes rares sont à l'inverse plus fréquemment isolés en période hivernale. L'effet saisonnier paraît plus marqué chez les multipares, mais les variations observées sont similaires.

\section{d / variation interannuelle}

L'étude s'étant déroulée du 01/02/86 au $31 / 01 / 90$, l'échantillon est divisé en 4 périodes de 12 mois, du $1^{\text {er }}$ février au 31 janvier de l'année suivante (années 1 à 4). Il existe un effet très significatif de l'année pour l'ensemble des germes isolés $(\mathrm{P}<0,001)$. L'augmentation de l'incidence des infections intramammaires associées à des germes mineurs en année 2 (figure 6) est principalement due aux staphylocoques (AS). Les bacillus (AB) étant plus fréquents en année 3 . En revanche, les germes pathogènes majeurs diminuent régulièrement au cours des 4 années, passant de 26,1 à $16,7 \%$. Cette dimi-
Figure 6. Variation annuelle des infections intramammaires.

Fréquence (\%)

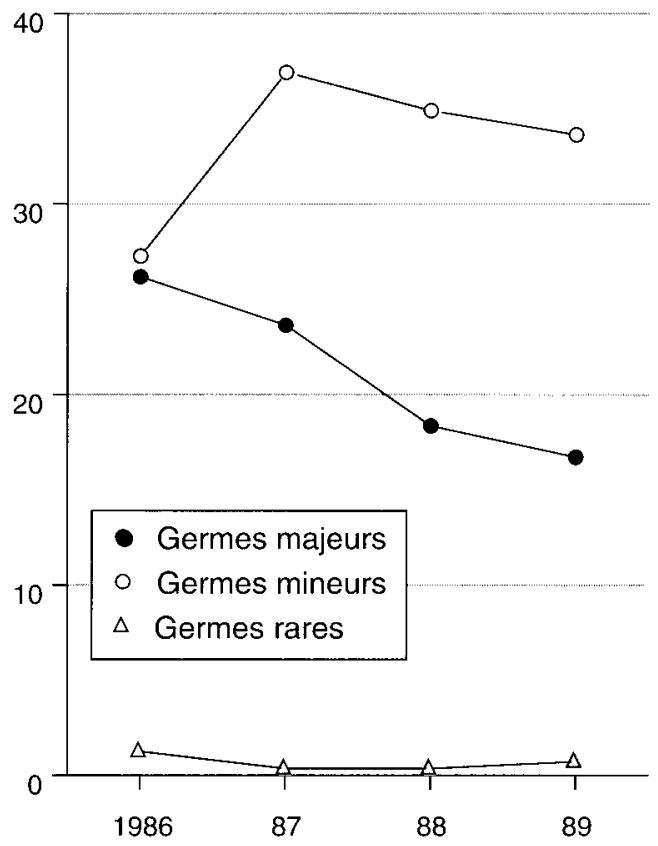

Figure 7. Variation annuelle et par rang de lactation des infections intramammaires dues aux germes pathogènes majeurs.

Fréquence annuelle (\%)

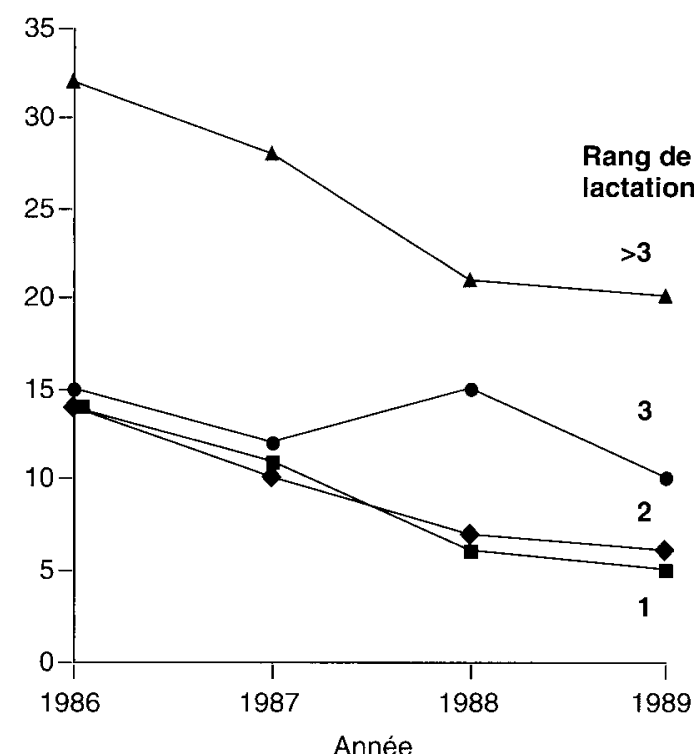

nution relève d'un effet cumulé de la diminution de tous les types de germes, bien que d'une façon moins marquée pour $S$. uberis et surtout $E$. coli. Les germes rares diminuent également: $1,3 \%$ (année 1) à $0,3 \%$ (année 3 ) puis $0,7 \%$ (année 4). La sensible remontée de la fréquence en année 4 est due à C. albicans. L'étude par rang de lactation montre cependant que la diminution progressive des fréquences est plus marquée pour les rangs $>3$, en particulier pour les germes pathogènes majeurs (figure 7). 
Figure 8. Variations départementales des infections intramammaires dues aux pathogènes rares.

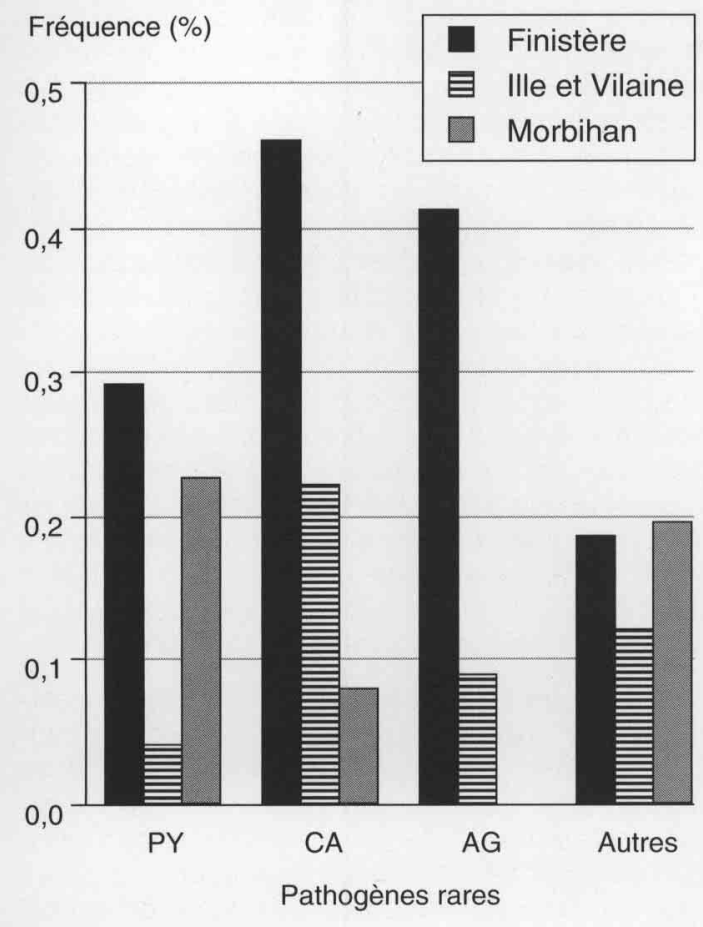

\section{e / variation interdépartementale}

Il y a plus de germes pathogènes mineurs dans les 13 exploitations du Finistère (près de 3 fois plus que dans les deux autres départements - Morbihan et Ille-et-Vilaine : $68,7 \%$ vs 28,4 et 17,2 respectivement). Cette différence se retrouve pour tous les types de germes, mais plus particulièrement pour AS. Les germes pathogènes majeurs sont également plus fréquemment isolés dans les exploitations du Finistère : $36,5 \%$ vs 20,9 pour le Morbihan et 13,7 pour l'Ille-et-Vilaine. L'écart est plus marqué pour les Streptocoques (AC) et $S$. aureus (ST) et, dans une moindre mesure, pour $E$. coli (EC) qui affecte plus souvent qu'ailleurs les vaches de rang de lactation $>3$. Enfin, les germes rares sont plus fréquents dans les exploitations du Finistère (figure 8 ) : $1,3 \%$ vs $0,5 \%$ en Ille et Vilaine et dans le Morbihan. Considérant tous les types de germe, seuls $20,2 \%$ des prélèvements réalisés dans le Finistère sont stériles, vs $39,2 \%$ en Ille-etVilaine et $49,9 \%$ dans le Morbihan.

\section{6 / Mammites cliniques et infections intramammaires}

Sur 4164 prélèvements non-stériles, 1855 (soit $44,5 \%$ ) ont été précédés ou suivis d'au moins une mammite clinique au cours de la lactation. Cette fréquence est de $29,7 \%$ pour les lactations présentant un prélèvement stérile.

Dans les analyses ultérieures, seules les mammites cliniques survenant au cours des deux premiers mois de lactation ont été prises en compte, les prélèvements de lait ayant eu lieu en début de lactation. Dans ces conditions la fréquence observée chez les vaches*lacta-
Figure 5. Evolution saisonnière des fréquences d'infections intramammaires.

Fréquence (\%)

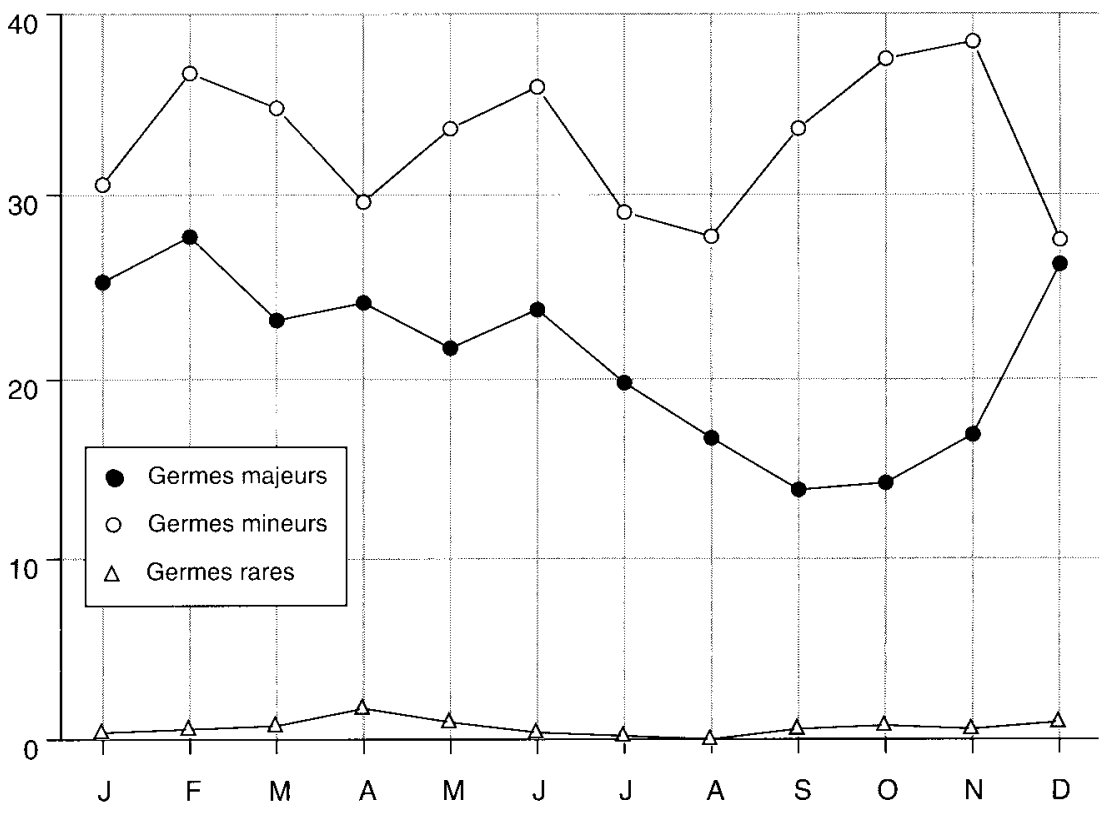

Fréquence $(\%)$

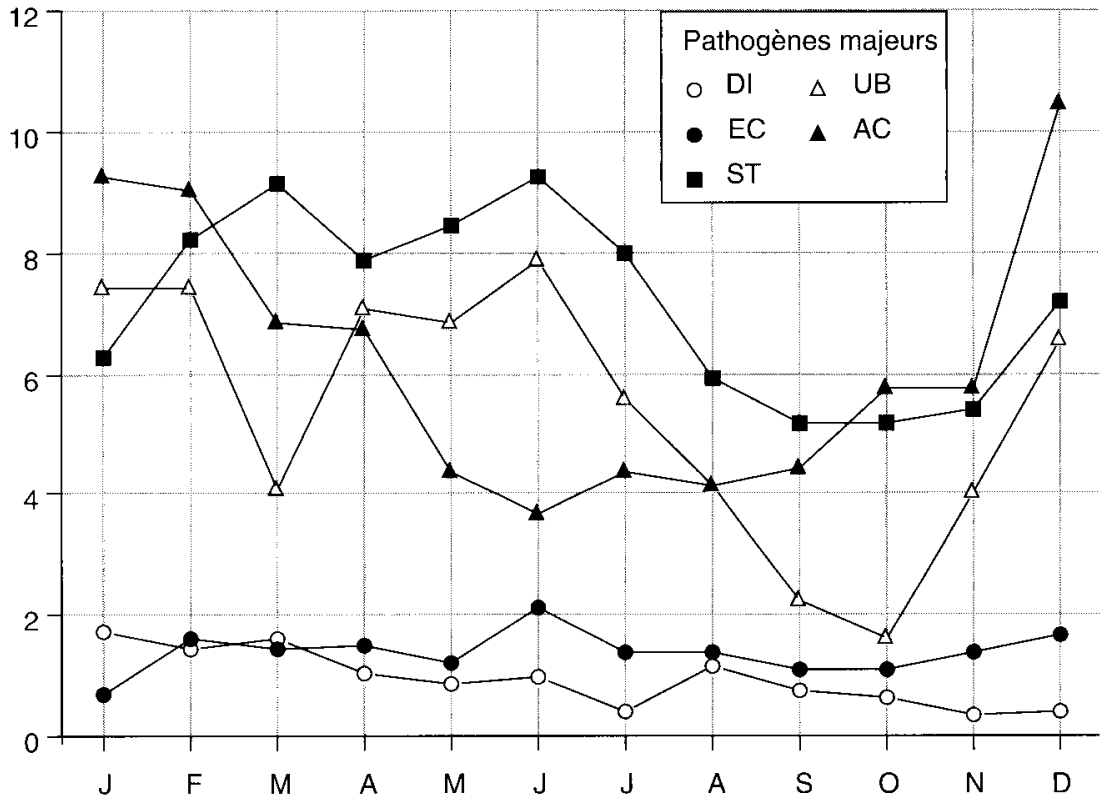

tions présentant un germe majeur est de $20,4 \%$ vs $10,9 \%$ (germes mineurs), $35,3 \%$ (germes rares), $11,55 \%$ (absence de germes). Ainsi, il n'y a pas de différence entre les lactations indemnes d'infections intramammaires et celles affectées par de germes mineurs. On observe une plus forte incidence des germes mineurs lorsque surviennent des mammites avec signes inflammatoires (MEIN) ou non précisés (MEXX) (tableau 7). Les germes majeurs sont plutôt associés aux mammites de type MELM (inflammation + lait modifié). Les mammites avec signes généraux (MEFI) sont proportionnellement plus nombreuses chez les vaches ayant présenté un prélèvement stérile. La prédominance des germes majeurs et rares s'affirme lorsqu'on considère chaque germe 
Tableau 7. Taux d'incidence (\%) des différents types de mammite clinique en fonction des types de germes isolés en début de lactation (et fréquence rapportée selon le type de mammite considéré).

\begin{tabular}{|l|ccc|c|}
\hline Type de & \multicolumn{3}{|c|}{ Type de germes pathogènes } & Absence \\
mammites & GMIN & GMAJ & GRAR & de germes \\
\hline MELA & 4,17 & 8,83 & 5,88 & 5,12 \\
& $(24,3)$ & $(32,8)$ & $(0,7)$ & $(42,2)$ \\
MEIN & 1,38 & 1,92 & 3,92 & 1,17 \\
& $(31,8)$ & $(28,3)$ & $(1,7)$ & $(38,0)$ \\
MELM & 1,80 & 4,26 & 7,84 & 2,06 \\
& $(23,7)$ & $(35,8)$ & $(2,0)$ & $(38,4)$ \\
MEXX & 3,18 & 4,74 & 13,7 & 2,55 \\
& $(31,6)$ & $(30,0)$ & $(2,6)$ & $(35,7)$ \\
MEFI & 0,8 & 2,04 & 3,92 & 1,36 \\
& $(19,6)$ & $(31,7)$ & $(1,8)$ & $(46,7)$ \\
MEGA & 0 & 0 & 0 & 0,03 \\
& $(0)$ & $(0)$ & $(0)$ & $(100)$ \\
\hline
\end{tabular}

Tableau 8. Fréquence des mammites cliniques (en \%) au cours des deux premiers mois de lactation selon les germes pathogènes isolés.

\begin{tabular}{|l|c|}
\hline Germe isolé (groupe) & Fréquence \\
\hline AS (GMIN) & 11,38 \\
Absence de germes & 11,55 \\
BO (GMIN) & 11,56 \\
AC (GMAJ) & 13,04 \\
AB (GMIN) & 15,01 \\
ST (GMAJ) & 19,20 \\
EC (GMAJ) & 28,30 \\
UB (GMAJ) & 31,67 \\
DI (GMAJ) & 32,88 \\
Autres (GRAR) & 35,29 \\
\hline
\end{tabular}

Tableau 9. Evolution des fréquences (\%) de mammites cliniques selon les classes de quantité des germes pathogènes isolés.

\begin{tabular}{|c|c|}
\hline Classe de quantité & Fréquence \\
\hline Pathogènes majeurs & \\
ST1 & 21,5 \\
ST2 & 20,7 \\
ST3 & 21,8 \\
UB1 & 15,2 \\
UB2 & 39,3 \\
UB3 & 39,6 \\
DI1 & 25,0 \\
DI2 & 30,4 \\
D13 & 42,8 \\
EC1 & 22,4 \\
EC2 & 30,0 \\
EC3 & 47,1 \\
AC1 & 14,6 \\
AC2 & 18,7 \\
AC3 & 19,4 \\
\hline AB1 & \\
AB2 & 16,3 \\
AB3 & 10,0 \\
AS1 & 14,8 \\
AS2 & 12,8 \\
AS3 & 11,6 \\
BO1 & 12,1 \\
BO2 & 15,4 \\
BO3 & 13,2 \\
\hline
\end{tabular}

(tableau 8). En ne considérant que les germes majeurs les plus fréquents (ST et UB) on observe une plus forte incidence des mammites de type MEIN ou MEFI lors d'infections intramammaires dues à $S$. uberis, et de type MELA avec $S$. aureus. La fréquence des mammites cliniques a tendance à augmenter selon les classes de quantité de germes (tableau 9), en particulier lorsqu'il s'agit de germes pathogènes majeurs, à l'exception toutefois de $S$. aureus. Il y a une différence significative du taux d'incidence des mammites chez les animaux présentant un seul germe majeur $(23,5 \%)$ par rapport aux animaux en ayant présenté deux $(27,6 \%)$. Il n'en est pas de même pour les germes mineurs $(10,4 \%$ vs $12,9 \%)$. L'association GMAJ * GRAR se traduit par une fréquence élevée de mammites cliniques $(28,5 \%)$ de même que l'association GMIN * GRAR $(35,7 \%)$. L'association GMIN*GMAJ donne un résultat intermédiaire $(24,5 \%)$.

Seules les associations entre germes spécifiques les plus couramment observées ont pu être testées. Ainsi, l'association ST * UB se traduit par une fréquence de $28 \%$ de mammites cliniques. Elle est de $30,7 \%$ avec l'association $\mathrm{ST}^{*} \mathrm{DI}$ et de $5,2 \%$ seulement avec l'association $\mathrm{AB}^{*} \mathrm{AC} * \mathrm{AS}$. Par type de mammites, les occurences devenant faibles, nous n'avons testé que les associations les plus courantes. Ainsi, la fréquence des mammites de type MEFI est plus importante avec une association de deux germes majeurs $(4,6 \%)$ qu'avec un seul $(2,8 \%)$.

\section{7 / Concentrations cellulaires et infections intramammaires}

Les prélèvements contenant un germe pathogène mineur proviennent de vaches qui, lors des deux premiers contrôles, ont dépassé un taux de 400000 cellules dans $17,8 \%$ des cas. Ce pourcentage est de $43,3 \%$ pour un germe majeur, de $42,8 \%$ pour un germe rare, de $15,2 \%$ en cas d'absence de germes. Pour les germes majeurs, les taux les plus élevés sont surtout observés avec $S$. uberis (tableau 10). La présence de germes rares est associée à des taux supérieurs à 800000 cellules (généralement $20 \%$ ces contrôles). La présence simultanée de deux germes pathogènes majeurs dans un même prélèvement n'augmente pas la pro-

Tableau 10. Pourcentages des deux premiers contrôles supérieurs à 800000 cellules et compris entre 400000 et 800000 selon les germes isolés dans le lait de traite.

\begin{tabular}{|c|c|c|}
\hline $\begin{array}{l}\text { Germes } \\
\text { pathogènes }\end{array}$ & $\begin{array}{c}\text { supérieurs à } \\
\mathbf{8 0 0 ~ 0 0 0}\end{array}$ & $\begin{array}{c}\mathbf{4 0 0} \mathbf{0 0 0} \\
\mathbf{a ̀ ~} \mathbf{8 0 0 0 0 0}\end{array}$ \\
\hline Majeurs & & \\
UB & 36,9 & 26,6 \\
DI & 28,7 & 31,5 \\
EC & 27,3 & 16,9 \\
ST & 20,0 & 22,2 \\
AC & 14,6 & 10,7 \\
\hline Mineurs & & \\
AS & 8,4 & 9,8 \\
AB & 7,9 & 6,9 \\
\hline
\end{tabular}


babilité d'observer des taux cellulaires supérieurs à 400000 cellules $(54,2$ vs $56,1 \%)$. En revanche, les taux sont sensiblement plus élevés dans les laits contenant un seul germe mineur $(21,1 \%)$ que dans les prélèvements où 2 germes mineurs ont été isolés $(15,2 \%)$. L'association GMIN * GMAJ entraine des fréquences intermédiaires $(36,2 \%)$. La fréquence des mammites augmente régulièrement avec le niveau de concentration cellulaire dans le lait de facon parallèle pour les germes mineurs et pour les germes majeurs (figure 9). On n'observe pas de différence significative entre les animaux présentant un germe mineur et les animaux sans germes.

\section{3 / Discussion}

La littérature concernant les infections intra-mammaires est très abondante, mais, en règle générale, les études publiées portent moins sur un dépistage systématique des germes pathogènes présents à un instant donné dans la mamelle que sur les germes responsables des mammites cliniques. Il est donc difficile de comparer la hiérarchie des fréquences des germes observées dans la présente étude avec celle publiée par d'autres auteurs (Barlett et al 1992, par exemple). Certains germes pathogènes n'ont plus l'importance qu'ils avaient il y a quelques années. C'est le cas de $S$. agalactiae, isolé dans $0,13 \%$ de nos prélèvements alors qu'il était observé dans plus de 10\% des prélèvements il y a 10-15 ans (Funk et al 1992, Agger et al 1994). Globalement, en dépit de la forte variabilité du taux de présence des bactéries pathogènes d'une étude à l'autre, il ressort que $S$. aureus, les streptocoques (S. uberis, S. dysgalactiae) et $E$. coli demeurent les germes majeurs dominants dans la plupart des enquêtes récentes. Dans les échantillons de lait prélevés au moment du tarissement, Schultze (1983) observe une hiérarchie similaire : autres staphylocoques ( $64 \%$ des prélèvements non-stériles vs $52 \%$ dans notre étude), S. aureus $(14,7 \%$ vs $13,2 \%)$, autres streptocoques $(7,3 \%$ vs $12,3 \%), S$. uberis $(6,7 \%$ vs $10,1 \%)$, E. coli $(4,7 \%$ vs $2,5 \%), S$. dysgalactiae ( $1,3 \%$ vs $1,7 \%)$ et $S$. agalactiae $(0,7 \%$ vs $0,2 \%)$. Les bacillus autres que cereus sont considérés par certains auteurs comme des germes de contamination. Cependant, la fréquence observée dans notre échantillon $(3,5 \%)$ est comparable à celle reportée $(3,1 \%)$ par Sharma et al (1993). L'ensemble des auteurs s'accorde pour considérer que les infections intramammaires sont plus fréquentes en début de lactation. Le premier mois représente la période la plus sensible (Schukken 1990). Les coliformes (notamment $E$. coli) sont cependant plus stables que les streptocoques qui diminuent plus rapidement conformément à nos résultats (Larry Smith et al 1985). A l'inverse, les mêmes auteurs relèvent une augmentation significative des infections dues à $C$. bovis au cours de la lactation, ce qui corrobore nos résultats. L'effet du rang de lactation est bien observé par de nombreux auteurs. Les caractéristiques physiques de la mamelle des vaches âgées (perte
Figure 9. Evolution des fréquences de mammites cliniques selon les niveaux de concentrations cellulaires et les types de germes.

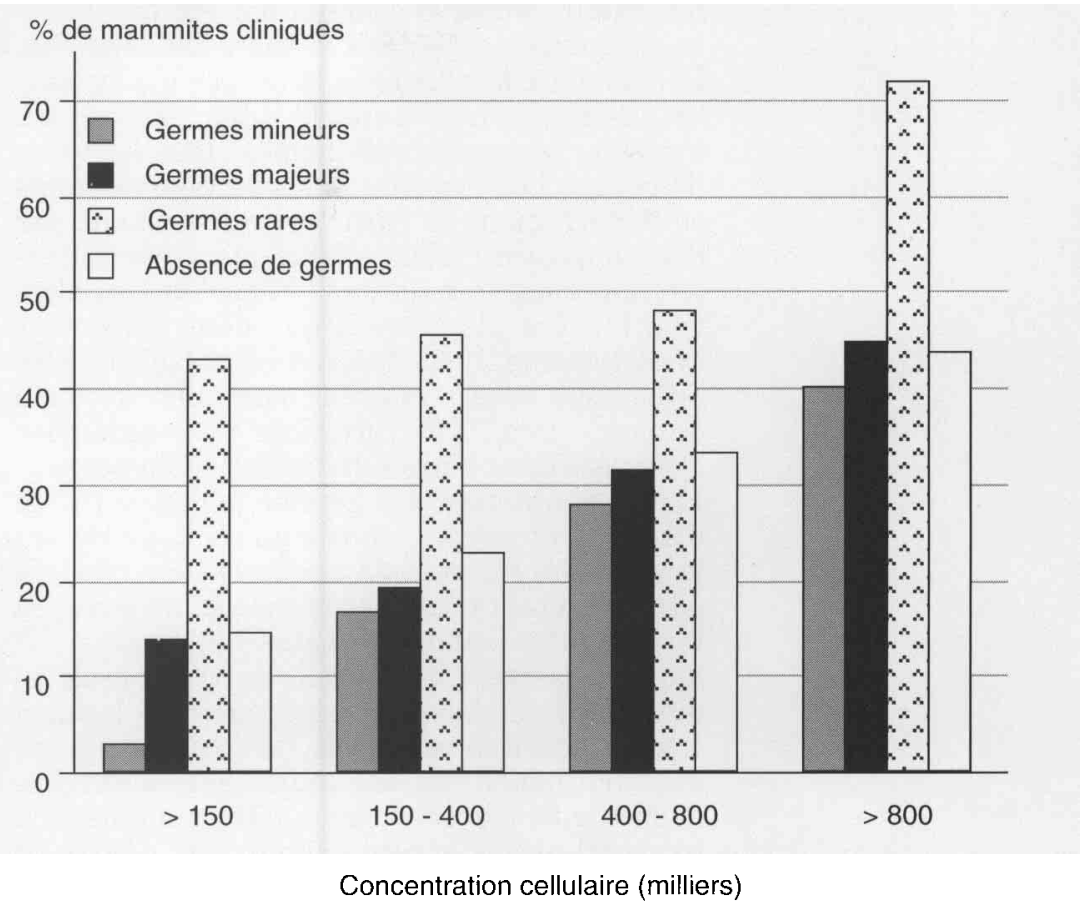

d'élasticité du tissu mammaire qui contribue à la diminution de la distance entre les trayons et le sol, augmentation de la perméabilité du sphincter ...) contribuent à l'augmentation des fréquences avec le rang de lactation et cet aspect aurait été amplifié sans l'élimination de vaches mammiteuses. Cependant, les staphylocoques coagulase- (pathogènes mineurs) sont moins sensibles à l'effet de l'âge (Smith et Hagstad 1986), ce qui explique sans doute la plus forte incidence observée dans notre étude des staphylocoques chez les primipares. L'effet saisonnier est observé de façon contradictoire du fait de l'imbrication des stades physiologiques et du rythme saisonnier. Cependant, les prélèvements ayant été réalisés, dans notre étude, en fonction du stade physiologique des animaux, les variations mensuelles sont indépendantes de l'effet stade. La diminution des fréquences pendant la période estivale, avec une sensible augmentation au mois de juin a déjà été observée pour $E$. coli et $S$. aureus (Wilesmith et al 1986, Schukken 1988). De même, le pic estival (août) observé pour $S$. dysgalactiae a été mentionné par Wilesmith et al (1986). D'après Smith et Hagstad (1986), l'incidence des infections intramammaires dues à $S$. aureus présente un pic au mois de mars, puis à l'automne. Dans notre échantillon, nous observons une évolution similaire, mais plus particulièrement pour les vaches multipares de rang de lactation égal à 3 . En revanche, ces mêmes auteurs ne trouvent pas d'effet saisonnier associé à la présence de germes pathogènes mineurs. L'action défavorable de la période hivernale (confinement des animaux, saison de vêlage, conditions climatiques ...) sur la santé de la mamelle et l'augmentation des germes pathogènes, notamment d'origine environnementale est bien connue (Francis 1985). Les différences observées entre les départements peuvent paraître surprenantes. 
Pourtant cet "effet département" ne peut être imputé à des différences dans les analyses de laboratoire puisque celles-ci ont été préalablement harmonisées dans le cadre de l'enquête. Par ailleurs, la plus forte incidence d'infections intramammaires dans le département du Finistère concerne pratiquement tous les types de germe. Les exploitations sélectionnées dans ce département se caractérisent souvent par des troupeaux à faible effectif, mais à forte densité dans les stabulations, et par une production laitière plus élevée que dans les autres départements. Il est donc vraisemblable que les différences notées relèvent d'un effet "système de production". Les variations inter-annuelles observées dans notre échantillon, et en particulier la diminution des germes majeurs, relève d'un effet propre à l'enquête au cours de laquelle plusieurs facteurs de risque ont été corrigés par les éleveurs. Les germes isolés ne sont pas forcément responsables des mammites cliniques observées, l'intervalle entre les prélèvements et la maladie pouvant varier de 1 jour à 7 semaines. Cependant, les germes pathogènes majeurs demeurent des indicateurs d'une fréquence plus élevée de mammites cliniques, en particulier les streptocoques (S. uberis et S. dysgalactiae). Dans une enquête réalisée dans le Sud-Ouest, Fabre et al (1991), notent que $23 \%$ des mammites cliniques étaient associées à $S$. uberis. En revanche, $E$. coli est souvent isolée dans les laits mammiteux tant en France (29\% selon Fabre et al 1991) qu'à l'étranger (14,6\% selon Barlett et al 1992), mais sa fréquence est plus rare dans les infections intramammaires (1\% selon Barlett et al 1992 ; 1,35\% dans notre étude). Ceci s'explique par la faible persistance d'E. coli dans la mamelle soumise à un traitement antibiotique. La présence d'un germe pathogène mineur ne constitue pas un indicateur des mammites cliniques, puisque, au contraire, les fréquences sont plus faibles que chez les vaches sans germes en début de lactation. Matthews et al (1991) ont d'ailleurs montré que la prévalence des nouvelles infections mammaires dans les quartiers non-infectés était deux fois plus élevée que dans les quartiers contenant un germe pathogène mineur (staphylocoque coagulase-). Selon Rainard et Poutrel (1988), la fréquence des nouvelles infections est divisée par deux lors d'une infection pré-existante par un germe pathogène mineur, le plus souvent un staphylocoque coagulase-, mais aussi $C$. bovis. D'ailleurs, nous constatons plus de mammites aiguës (MEFI) chez les vaches ayant eu un prélèvement de lait stérile. La plus grande sensibilité des vaches présentant un germe pathogène majeur à une augmentation des concentrations cellulaires a bien été décrite. Selon Serieys (1985), la concentration cellulaire passe de 50000 cellules $/ \mathrm{ml}$ en l'absence d'infection à 100000 en cas d'infection par un pathogène mineur, et à 500000 environ en cas d'infection par un pathogène majeur. Dans notre étude, les fréquences de taux $>400000$ les plus élevées sont rencontrées chez les vaches infectées par $S$. uberis. Fabre et al (1991) isolent $S$. uberis dans $17 \%$ des cas de mammites subcliniques contre $29 \%$ pour $S$. aureus, soit selon une hiérarchie différente de la notre. Enfin, il subsiste une forte variabilité des fréquences observées dans les exploitations. Cette variabilité est bien entendu le reflet des conditions d'hygiène et de management. C'est en s'appuyant sur ces différences observées, que nous comptons mettre en évidence les facteurs de risque des infections intramammaires.

\section{Conclusion}

Environ une vache sur deux abrite dans sa mammelle un germe pathogène. Ce résultat est conforme à ceux publiés par d'autres auteurs dans les pays de l'hémisphère Nord (Barlett et al 1992). Plus de 30\% d'entre eux sont des pathogènes majeurs qui entraînent souvent des mammites cliniques ou subcliniques. La présence de ces germes relève de caractéristiques propres aux animaux, mais surtout de pratiques d'élevage (pratiques de traite, hygiène générale, alimentation, conditions de logement ...) sur lesquelles l'éleveur et son environnement technique peuvent intervenir efficacement. C'est à la mise en évidence de ces facteurs que nous allons maintenant nous attacher dans le cadre du dépouillement de la base de données GWEN-HA-DU.

\section{Remerciements}

Les prélèvements de lait ont été assurés par les techniciens des services vétérinaires du Finistère (Mrs Azou, Goaper et Maguet), du Morbihan (Mrs Bodiguel et Le Men) et d'Ille-etVilaine (Mrs Garaud, Dagorne, Renaud et Bouliou). Nous remercions également Mrs Serieys et David qui ont participé à la formation des techniciens pour réaliser les prélèvements dans les meilleures conditions, et les responsables des laboratoires départementaux qui ont mené à bien l'ensemble des analyses microbiologiques. Enfin, nous remercions tous ceux qui de près ou de loin (éleveurs, praticiens, vétérinaires-inspecteurs) ont contribué au succès de cette étude. Le dépouillement des données sur l'aspect "qualité du lait" a bénéficié du concours financier de l'Association GALA.

\section{Références bibliographiques}

Agger J.F., Priou C., Huda A., 1994. Risk factors for transmission of $\mathrm{S}$. agalactiae infection between Danish dairy herds : a case control study. Actes du Coll. Int. d'Ecopathologie et Gestion de la Santé Animale. ClermontFerrand, 18-20 Octobre 1993. Vet. Res. (sous presse).

Barlett P.C., Miller G.Y., Lance S.E., Heider L.E., 1992. Clinical mastitis and intramammary infections on Ohio dairy farms. Prev. Vet. Med., 12, 59-71.
Barnouin J., Fayet J.C., Brochart M., Bouvier M., 1993. Enquête écopathologique continue : 1 . Hiérarchie de la pathologie observée en élevage bovin laitier. Ann. Rech. Vet., 14, 247-252.

Coulon J.B., Landais E., Garel J.P., 1989. Alimentation, pathologie, reproduction et productivité de la vache laitière. INRA Prod. Anim., 2(3), 171-188. 
Fabre J.M., Berthelot X., Blanc M.C., Blanc M.F., Lebret P., 1991. Estimation de la fréquence des différents germes responsables d'infections mammaires en élevage bovin laitier dans le Sud Ouest de la France. Rev. Med. Vet., 142, 11, 823-829.

Faye B., Barnouin J., 1987. Motivations et objectifs de l'enquête écopathologique Bretagne. Bull. Tech. C.R.Z.V. Theix, INRA, 69, 9-13.

Faye B., Dorr N., Lescourret F., Barnouin J., Chassagne M., 1993. Les pratiques d'élevage associées au "complexe atteinte de la mamelle". Actes du Coll. Int. d'Ecopathologie et Gestion de la Santé Animale. Clermont-Ferrand, 18-20 Octobre 1993. Vet. Res. (sous presse).

Francis P.G., 1985. An approach to the herd mastitis problem. Brit. Vet. J., 140, 22-26.

Funk D.A., Berger P.J., Freeman A.E., 1982. Environmental and physiological factors at drying off and post calving. J. Dairy Sci., 65, 1258-1268.

Larry Smith K., Todhunter D.A., Schoenberger P.S., 1985. Environmental effects on cow health and performance. J. Dairy Sci., 68, 1532-1553.

Lescourret F., Barnouin J., Chassagne M., Faye B., Genest M., 1993. Data modelling for database design in production and health monitoring systems for dairy herds. J. Dairy Sci., 76, 1053-1062.

Matthews K.R., Harmon R.J., Langlois B.E., 1991. Effect of naturally occuring coagulase-negative staphylococci infections on new infections by mastitis pathogens in the bovine. J. Dairy Sci., 74, 1855-1859.

Pluvinage P., Ducruet T., Josse J., Monicat F., 1991. Facteurs de risque des mammites des vaches laitières. Résultats d'enquête. Rec. Med. Vet., 167, 105-112.
Poutrel B., 1985. Généralités sur les mammites de la vache laitière : processus infectieux, épidémiologie, diagnostic, méthodes de contrôle. Rec. Med. Vet., 161, 497-511.

Rainard P., Poutrel B., 1988. Effect of naturally occuring intramammary infections by minor pathogens on new infections by major pathogens in cattle. Am. J. Vet. Res., 49, 327-329.

Schukken Y.H., Erb H.N., Sears P.M., Smith R.D., 1988. Ecologic study of the risk factors for environmental mastitis in cows. Am. J. Vet. Res., 49, 766-771.

Schukken Y.H., 1990. Epidemiological studies on clinical mastitis in dairy herds with a low bulk somatic cell count. Thèse Doct Univ, Utrecht, 158p

Schultze W.D., 1983. Effects of a selective regimen of dry cow therapy on intramammary infection and on antibiotic sensitivity of surviving pathogens. J. Dairy Sci., 66, 892-903

Serieys F., 1985. Concentration cellulaire du lait individuel de vache : influence de l'état d'infection mammaire, du numéro, du stade de lactation et de la production laitière. Ann. Rech. Vet., 16, 255-261.

Sharma M., Katoch R.C., Nagal K.B., Sambyal D.S., 1993. Studies on the prevalence of mastitis in an organised dairy farm at Palampur, Pradesh. Indian J. Dairy Science, 46(1) 37-38.

Smith R.E., Hagstad H.V., 1986. Infection of the bovine udder with coagulase negative staphylococci. Prev. Vet. Med., 35-43.

Wilesmith J.W., Francis P.G., Wilson C.D., 1986. Incidence of clinical mastitis in a cohort of british dairy herds. Vet. Rec., 118, 199-204.

\section{Summary}

Intramammary infections of dairy cows in ecopathological survey in Britain.

In the frame of an four-year ecopathological study in 47 intensive dairy farms from Britain (France), a milk simple for bacteriological analysis has been done on each cow at the beginning of the lactation. As the whole, 7852 analysis have been realized. The purpose of the present paper is to describe the results of these analysis and to study some factors as space and time.

$53 \%$ of the samples contain pathogen germs. Minor pathogens (Staphylococcus coagulase-, bacillus, C. bovis) are dominant, but major pathogens (S. aureus, S. uberis, S. dysgalactiae, E. coli, others streptococcus) are present in one third of non-sterile samples. Milk has been samples in a intervalle of 3 days to 7 weeks after calving. This does not get an important bias, because the variation of the rate is not significant.
Intramammary infections (IMI) due to major pathogens increase with the parity. The winter time is defavourable, but there is a higher in june for major pathogens. There is a year effect, the rate of IMI having a trend to decrease. Finistère is more affected (36.5\% of IMI with major pathogens) than Morbihan (20.9\%) and Ille-et-Vilaine (13.7\%).

If we consider only early mastitis and two first milking controle (Somatic cell count-SCC-registration), we observe a higher incidence of mastitis $(20.4 \%)$ and SCC > $4 \times 106(43.3 \%)$ with major pathogens than with minor (respectively 10.9 and $17.8 \%$ ).

The rule of farming pratices and environmental conditions will be taken in account in a second step to determine the risk factors of IMI.

FAYE B., DORR Nelly, LESCOURRET Françoise, BAR NOUIN J., CHASSAGNE Michelle, 1994. Les infections intra-mammaires chez la vache laitière dans l'enquête écopathologique Bretagne. INRA Prod. Anim., 7 (1), 55-65. 